\title{
Galois Group at Each Point for Some Self-Dual Curves
}

\author{
Hiroyuki Hayashi, ${ }^{1}$ and Hisao Yoshihara ${ }^{2}$ \\ ${ }^{1}$ Graduate School of Science and Technology, Niigata University, Niigata 950-2181, Japan \\ ${ }^{2}$ Department of Mathematics, Faculty of Science, Niigata University, Niigata 950-2181, Japan
}

Correspondence should be addressed to Hisao Yoshihara; yosihara@math.sc.niigata-u.ac.jp

Received 10 October 2012; Accepted 21 December 2012

Academic Editor: Michel Planat

Copyright (c) $2013 \mathrm{H}$. Hayashi and H. Yoshihara. This is an open access article distributed under the Creative Commons Attribution License, which permits unrestricted use, distribution, and reproduction in any medium, provided the original work is properly cited.

We study the Galois group defined by a point projection for plane curve. First, we present a sufficient condition that the group is primitive and then determine the structure at each point for some self-dual curves.

\section{Introduction}

This study is a continuation of [1-4], and so forth. In general, it is not easy to determine the Galois group $G_{P}$ at every point $P$ for plane curve, in particular for curve with singular point. When we determine the structure of $G_{P}$, it is important to know whether it is primitive or not. However, there are not so many results which are useful for our purpose (cf. [5]). In this paper we give a geometrical criterion and then determine the group at each point for some self-dual curves.

Let $k$ be an algebraically closed field of characteristic zero. We fix it as the ground field of our discussions. Let $C$ be an irreducible plane curve of degree $d(\geq 2)$ and $K=k(C)$ the rational function field of $C$. Let $(X: Y: Z)$ be a set

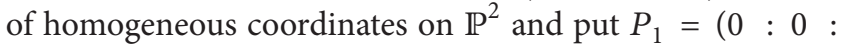
$1), P_{2}=(0: 1: 0)$, and $P_{3}=(1: 0: 0)$. Let $F(X, Y, Z)$ be the defining equation of $C$ and put $f(x, y)=F(X, Y, Z) / Z^{d}$, where $x=X / Z, y=Y / Z$.

1.1. Galois Group. Let $r: \widetilde{C} \rightarrow C$ be the resolution of singularities of $C$. For a point $P \in \mathbb{P}^{2}$, let $\widehat{P}$ be the dual line in the dual space $\widehat{\mathbb{P}^{2}}$ of $\mathbb{P}^{2}$ corresponding to $P$. We define the morphism $\pi_{P}$ by

$$
\pi_{P}: \widetilde{C} \ni Q \longmapsto \widehat{\ell_{P R}} \in \widehat{P} \cong \mathbb{P}^{1},
$$

where $\widehat{\ell_{P R}}$ is the point in $\widehat{\mathrm{P}^{2}}$ corresponding to the line $\ell_{P R}$, which passes through $P$ and $R=r(Q)$ if $P \neq R$. In case $P=$ $R$, the line $\ell_{P R}$ is the tangent line to the branch of $C$ at $R$.
Clearly, we have $\operatorname{deg} \pi_{P}=d-m_{P}(C)$ and a field extension $\pi_{P}^{*}$ : $k\left(\mathbb{P}^{1}\right) \hookrightarrow K=k(\widetilde{C})$, where $m_{P}(C)$ denotes the multiplicity of $C$ at $P$. In case $P \notin C$, we understand $m_{P}(C)=0$. We put $n(P)=d-m_{P}(C)$; if there is no fear of confusion, we simply denote it by $n$. Since the extension depends only on $P$, we denote $k\left(\mathbb{P}^{1}\right)$ by $K_{P}$, that is, we have $\pi_{P}^{*}: K_{P} \hookrightarrow K$. Let $L_{P}$ be the Galois closure of $K / K_{P}$ and $G_{P}$ the Galois group $\operatorname{Gal}\left(L_{P} / K_{P}\right)$.

Definition 1. We call $G_{P}$ the Galois group at $P$ for $C$. In case $K / K_{P}$ is a Galois extension, the point $P$ is said to be a Galois point.

In case $k$ is the field of complex numbers, $G_{P}$ is isomorphic to the monodromy group of the covering $\pi_{P}: \widetilde{C} \rightarrow \mathbb{P}^{1}$ $[6,7]$.

\subsection{Self-Dual Curve}

Definition 2. A point $Q \in C$ is said to be a cusp of $C$ if it is a singular point and $r^{-1}(Q)$ consists of a single point. Furthermore, if $\mu: B_{Q}\left(\mathbb{P}^{2}\right) \rightarrow \mathbb{P}^{2}$ is a blow-up and $\mu^{-1}(Q)$ is a nonsingular point of the proper transform of $\mu^{-1}(C)$, the point $Q$ is said to be a simple cusp.

Denote by $\widehat{C}$ the dual curve of $C$.

Definition 3. If $\widehat{C}$ is projectively equivalent to $C$, then $C$ is said to be a self-dual curve. 
Suppose $C$ is smooth. Then, $C$ is self-dual if and only if $d=2$. However, if $C$ has a singular point, the condition that $C$ is self-dual becomes complicated. The following proposition has been known (cf. [8]).

Proposition 4. Suppose C is one of the following curves:

(1) C has just one singular point;

(2) $C$ is rational and has only simple cusps as singular points.

Then, $C$ is a self-dual curve if and only if $C$ is projectively equivalent to the curve defined by $y=x^{d}$.

Example 5. It seems that only a few self-dual curves have been known. Here, we present some of them

(I) $C_{(e, d)}$ : the curve defined by $Y^{e} Z^{d-e}=X^{d}, \operatorname{gcd}(e, d)=$ $1,1 \leq e \leq d-1 ;$

(II) $C_{(4)}$ : the curve defined by $\left(Y Z-X^{2}\right)^{2}=X^{3} Y$ (cf. [9]);

(III) $C_{54}$ : the curve defined by $(X Y-X Z+Y Z)^{3}+$ $54 X^{2} Y^{2} Z^{2}=0$ (cf. [10]).

For the curve $C_{(e, d)}$, if $1<e<d-1$, then $P_{1}=(0: 0: 1)$ and $P_{2}=(0: 1: 0)$ are not simple cusps and $C_{(e, d)}$ has no flex. The curve $C_{(4)}$ has two cusps $P_{1}$ and $P_{2}$, where $P_{1}$ is not a simple cusp. The curve $C_{54}$ has three cusps: $P_{1}, P_{2}$, and $P_{3}$ and the normalization is an elliptic curve. It is easy to find the dual curve of $C_{(e, d)}$; however, in the other curves we need some consideration, for the details, see $[9,10]$.

Remark 6. Let $\Phi_{C}$ be the rational map $\mathbb{P}^{2} \rightarrow \mathbb{P}^{2}$ giving the dual of $C$, that is,

$$
\Phi_{C}(X: Y: Z)=\left(\partial_{X} F: \partial_{Y} F: \partial_{Z} F\right)
$$

where $F$ is the defining equation of $C$. In the case where $C=$ $C_{(e, d)}$, the map $\Phi_{C}$ turns out to be a quadratic transformation of $\mathbb{P}^{2}$ :

$$
\Phi_{C}(X: Y: Z)=(-d Y Z: e Z X:(d-e) X Y) .
$$

We use the following notation:

(i) $Z_{m}$ : the cyclic group of order $m$;

(ii) $S_{d}$ : the symmetric group of degree $d$;

(iii) $i\left(X_{1}, X_{2} ; Q\right)$ : the intersection number of two curves $X_{1}$ and $X_{2}$ at $Q$;

(iv) $\ell_{P Q}$ : the line passing through $P$ and $Q$;

(v) $\ell_{P}$ : a line passing through $P$;

(vi) $T_{Q}=T_{Q}(C)$ : the tangent line to $C$ at $Q$.

\section{Statement of Results}

We need some preparations before stating the results. A curve means a nonsingular projective algebraic curve. Let $X_{1}$ and $X_{2}$ be curves and $f: X_{1} \rightarrow X_{2}$ a surjective morphism, which we call a covering for short. We denote by $e(R, f)$ the ramification index of $f$ at $R \in X_{1}$. If there is no fear of confusion, we simply denote it by $e(R)$.
Definition 7. Let $f: X_{1} \rightarrow X_{2}$ be the covering above. If there exists a curve $X_{3}$ and coverings $\alpha: X_{1} \rightarrow X_{3}$ and $\beta: X_{3} \rightarrow X_{2}$ such that $f=\beta \alpha, \operatorname{deg} \alpha \geq 2$ and $\operatorname{deg} \beta \geq 2$, then $f$ is said to be decomposable and $X_{3}$ an intermediate covering. If such a curve $X_{3}$ does not exist, then $f$ is said to be indecomposable (cf. [11]).

Definition 8. Let $f: X_{1} \rightarrow X_{2}$ be the covering above and $R_{1}, \ldots, R_{r}$ all the ramification points for $f$. Put $e\left(R_{i}\right)=e_{i}(1 \leq$ $i \leq r)$. The covering $f$ is said to be an $s$-covering over $f\left(R_{i}\right)$ if there exists no ramification point in $f^{-1} f\left(R_{i}\right)$ except $R_{i}$. The $f$ is said to be an $s$-covering if it is an $s$-covering over each $f\left(R_{i}\right)(1 \leq i \leq r)$.

Definition 9. With the same notation as in Definition 8, we call $\left\{\left(R_{1}, \ldots, R_{r}\right),\left(e_{1}, \ldots, e_{r}\right)\right\}$ (or, simply $\left.\left(e_{1}, \ldots, e_{r}\right)\right)$ the ramification data for $f$.

We give several sufficient conditions that $f$ is indecomposable. Some of them will not be used later in this paper.

Proposition 10. Let $f: X_{1} \rightarrow X_{2}$ be the covering above and $n=\operatorname{deg} f$. If one of the following conditions is satisfied, then $f$ is indecomposable.

(1) For some $i(1 \leq i \leq r), e_{i}$ is prime and $n<2 e_{i}$.

(2) $e_{1}=n-1$.

(3) $X_{2}$ is a rational curve, $f$ is an s-covering except over $f\left(R_{1}\right)$ and $e_{i}$ is prime for each $i \geq s+1$, where $f^{-1} f\left(R_{1}\right)=\left\{R_{1}, \ldots, R_{s}\right\}$.

Proposition 11. With the same notation as in Proposition 10, if $f$ is an s-covering and satisfies one of the following conditions, then $f$ is indecomposable.

(1) $X_{1}$ is a rational curve, $e_{1} \geq e_{2}, n-1 \geq e_{2}$, and $e_{i}$ is prime for each $i \geq 3$.

(2) $X_{1}$ is a rational curve and $e_{i}$ is prime for each $i \geq 2$.

(3) $X_{2}$ is a rational curve and $e_{i}$ is prime for each $i$.

Hereafter, we follow the notation in Section 1. By taking a suitable projective change of coordinates, we can assume the projection center is $P_{1}$ without changing the structure of $G_{P}$. Putting $y=t x$, we have $K_{P}=k(t)$ and $K=k(x, y)=k(t, x)$. Put $g(x)=f(x, t x) / x^{m} \in k(t)[x]$, where $m=m_{P}(C)$ and let $\left\{x_{1}, \ldots, x_{n}\right\}(n=n(P))$ be the roots of $g(x)=0$. Then, we can consider $G_{P}$ as a permutation subgroup of $S_{n}$. Note that $G_{P}$ is a transitive subgroup of $S_{n}$. Hence, $G_{P}$ is a primitive group if and only if the isotropy subgroup of an element of $\left\{x_{1}, \ldots, x_{n}\right\}$ is a maximal subgroup of $S_{n}$.

Theorem 12. The group $G_{P}$ is primitive if and only if $\pi_{P}$ is indecomposable. In particular, if $n(P)$ is a prime number, then $G_{P}$ is primitive for $P \in \mathbb{P}^{2}$.

Definition 13. Assume $Q \in C$ is a smooth point or a cusp. A line $\ell=\ell_{P Q}$ is said to be a simple $e$-tangent line to $C$ if the following conditions are satisfied: 
TABle 1

\begin{tabular}{cccccc}
\hline$P$ & $P_{1}$ & $P_{2}$ & $P_{3}$ & $P \in C \backslash\left\{P_{1}, P_{2}\right\}$ & $P \in \mathbb{P}^{2} \backslash C \cup\left\{P_{3}\right\}$ \\
$G_{P}$ & $Z_{d-e}$ & $Z_{e}$ & $Z_{d}$ & $S_{d-1}$ & $S_{d}$ \\
\hline
\end{tabular}

TABle 2

\begin{tabular}{cccc}
\hline$P$ & $P_{1}, P_{2}$ & $P \in C \backslash\left\{P_{1}, P_{2}\right\}$ & $P \in \mathbb{P}^{2} \backslash C$ \\
$G_{P}$ & $Z_{2}$ & $S_{3}$ & $S_{4}$ \\
\hline
\end{tabular}

(1) if $Q \neq P$ (resp., $Q=P$ ), then $i(C, \ell ; Q)=e$ (resp., $e+$ $m)$, where $e \geq 2$ and $m=m_{P}(C)$;

(2) the curves $C$ and $\ell$ have normal crossings except at $Q$.

Sometimes we call $\ell$ a simple $e$-tangent for short.

Note that a simple $e$-tangent $\ell_{P Q}$ yields an $s$-covering over $\pi_{P}(Q)$.

Lemma 14. We have the following assertions for $G_{P}$.

(1) If each line $\ell_{P}$ has normal crossings with $C$ or is a simple e-tangent line to $C$ such that e is a prime number, then $G_{P}$ is primitive (cf. [5, Lemma 4.4.4]).

(2) If there exists a simple 2-tangent line $\ell_{P}$, then $G_{P}$ contains a transposition.

The following lemma is well known.

Lemma 15. If a permutation group $G \subset S_{n}$ is primitive and contains a transposition, then it is a full symmetric group. lary.

Combining the results above, we get the following corol-

Corollary 16. If the covering $\pi_{P}: \widetilde{C} \rightarrow \mathbb{P}^{1}$ is one of the coverings in Propositions 10 or 11 and $\pi_{P}$ is an s-covering over $\pi_{P}\left(R_{i}\right)$ with $e_{i}=2$ for some $i(1 \leq i \leq r)$, then $G_{P}$ is a full symmetric group. In particular, if each line $\ell_{P}$ has normal crossings with $C$ or is a simple 2-tangent, then $G_{P}$ is a full symmetric group.

Corollary 16 implies [2, Theorem 1 and $1^{\prime}$ ]. Now we can state the structure of $G_{P}$ as follows.

Theorem 17. For the curves $C$ in Example 5, the Galois groups $G_{P}$ are as follows, where $Z_{1}$ indicates the trivial group

(I) the case $C=C_{(e, d)}$ (see Table 1$)$;

(II) the case $C=C_{(4)}$ (see Table 2);

(III) the case $\mathrm{C}=\mathrm{C}_{54}$ (see Table 3 ).

Remark 18. For the curves in Theorem 17, $P$ is a Galois point if and only if $G_{P}$ is a cyclic group. However, the same assertion does not hold true in general, see, for example, [3].

\section{Proofs}

First, we prove Propositions 10 and 11.
TABle 3

\begin{tabular}{lccc}
\hline$P$ & $P_{1}, P_{2}, P_{3}$ & $P \in C \backslash\left\{P_{1}, P_{2}, P_{3}\right\}$ & $P \in \mathbb{P}^{2} \backslash C$ \\
$G_{P}$ & $S_{3}$ & $S_{5}$ & $S_{6}$ \\
\hline
\end{tabular}

Claim 1. Suppose $f$ and a ramification point $R \in X_{1}$ satisfy the following conditions:

(1) $f$ is an $s$-covering over $f(R)$.

(2) $e(R)$ is prime.

If there exists an intermediate covering $\beta: X_{3} \rightarrow X_{2}$, then $\beta$ is unramified at $R^{\prime}=\alpha(R)$.

Proof. Suppose $\beta$ is ramified at $R^{\prime}$. Then, since $e(R)$ is prime, we have $e\left(R^{\prime}, \beta\right)=e(R, f)$, hence $R^{\prime}$ is not a branch point for $\alpha$. Then, there will appear another ramification point for $f$ in $f^{-1}(f(R))$. This is a contradiction.

The proof of Proposition 10 is as follows. Suppose $f$ is decomposable and there exists a covering $\beta: X_{3} \rightarrow X_{2}$ as in Definition 7. First, we prove the assertion (1). Since $e_{i}$ is prime, $\beta$ is unramified at $R_{i}^{\prime}$ by Claim 1 . Hence, we have $e\left(R_{i}, \alpha\right)=e\left(R_{i}, f\right)$. Since there exists at least two points in $\beta^{-1}\left(f\left(R_{i}\right)\right)$, we have $n=\operatorname{deg} f \geq 2 e\left(R_{i}, f\right)$, which contradicts the assumption. Next we prove (2). Clearly $\alpha$ and $\beta$ are ramified at $R_{1}$ and $R_{1}^{\prime}$, respectively. Put $B_{1}=f\left(R_{1}\right)$. Then, since $e_{1}=n-1, \beta^{-1}\left(B_{1}\right)$ consists of one or two points. In the former case, $\alpha^{-1}\left(\beta^{-1}\left(B_{1}\right)\right)$ consists of two points, on the other hand in the latter case $\alpha^{-1}\left(B_{1 i}\right)(i=1,2)$ consists of one point, where $\beta^{-1}\left(B_{1}\right)=\left\{B_{11}, B_{12}\right\}$. In each case we infer the inequality $n=\operatorname{deg} f \geq(n-1)+2$, which is a contradiction. We go to the proof of (3). Then, by Claim $1, B_{i}(i \geq 2)$ is not a branch point for $\beta$. Thus, $B_{1}$ is the only branch point for $\beta$. Then, by Hurwitz's formula, we have $2 g\left(X_{3}\right)-2=-2 b+c$, where $g\left(X_{3}\right)$ is the genus of $X_{3}, b$ is the degree of $\beta$, and $c \leq b-1$. Since $g\left(X_{3}\right) \geq 0$, this inequality implies $b=1$, which is a contradiction.

Next we prove Proposition 11. In each case we use the reduction to absurdity, that is, suppose $f$ is decomposable. So we use the notation $R_{i}^{\prime}=\alpha\left(R_{i}\right)(1 \leq i \leq r)$. In the case (I), by Claim 3, $\beta$ is unramified at $R_{i}^{\prime}(i \geq 3)$. Since $X_{2}$ and $X_{3}$ are rational, from Hurwitz's formula, we infer that $\beta$ is ramified with the index $e\left(R_{1}^{\prime}, \beta\right)=e\left(R_{2}^{\prime}, \beta\right)=\operatorname{deg} \beta$. Then, since there exists no ramification points in $f^{-1}\left(f\left(R_{i}\right)\right)$ except $R_{i}(i=$ 1,2), $\alpha$ must branch at $R_{1}^{\prime}$ and $R_{2}^{\prime}$. However, there exists an unramified point in $f^{-1}\left(f\left(R_{2}\right)\right)$, this is a contradiction. Therefore, $f$ is indecomposable. In the case (II), by Claim $1, \beta$ is unramified at $R_{i}^{\prime}$ for $i \geq 2$. Since $X_{3}$ is rational, by Hurwitz's formula, we have a contradiction. In the case (III) similarly, by Claim $1, \beta$ is unramified at every point; however, since $X_{2}$ is rational, $\beta$ must be an identity, which is a contradiction. This completes the proof of Proposition 11.

The proof of Theorem 12 is as follows: suppose $G_{P}$ is not primitive and let $G_{x}$ be the isotropy group of $x=x_{1}$ in $G_{P}$. Then, there exists a subgroup $H$ of $G_{P}$ such that $G_{x} \subsetneq H \subsetneq$ 
$G_{P}$. Let $C_{H}$ be the nonsingular model of the intermediate field which corresponds to $H$ by the Galois correspondence. Then, there exist the coverings $\alpha: \widetilde{C} \rightarrow C_{H}$ and $\beta: C_{H} \rightarrow \mathbb{P}^{1}$ such that $\pi_{P}=\beta \alpha$. Thus, $\pi_{P}$ is decomposable. The converse assertion is clear from the Galois correspondence.

The proof of Lemma 14 is simple. In view of Definition 13, we see that the assertion (1) is another expression of (3) in Proposition 11. The assertion (2) may be well known (cf. [7]).

Now we proceed to the proof of Theorem 17. The structure of $G_{P}$ depends on the covering $\pi_{P}$ and $\pi_{P}$ depends on the position of $P$. We prove by examining the cases where $P$ lies on the tangent line to $C$ at the cusp or at the flex. Hereafter, we assume $C$ is the curve in Theorem 17. Since $C$ is a self-dual curve and has only cusps as the singularity, the following remark is clear.

Remark 19. Suppose a line $\ell$ satisfies the following conditions:

(1) $\ell$ does not pass through any cusp;

(2) $\ell$ is not the tangent line to $C$ at the flex.

Then, $\ell$ is a simple 2 -tangent line to $C$ or $\ell$ and $C$ have normal crossings.

Proof of the Case (I). Assume $C=C_{(e, d)}$. It has the following property.

Claim 2. The tangent line $T_{P_{1}}$ (resp., $T_{P_{2}}$ ) is $Y=$ 0 (resp., $Z=0$ ) and $T_{P_{1}} \cap T_{P_{2}}=\left\{P_{3}\right\}$, which does not lie on $C$. In case $e=1$ (resp., $d-1), C$ has one flex at $P_{1}$ (resp., $P_{2}$ ). On the other hand, in case $1<e<d-1, C$ has no flex.

Proof. Calculating the Hessian of $X^{d}-Y^{e} Z^{d-e}$ (cf. [12]), we infer readily the assertions.

If $P=P_{1}, P_{2}$, or $P_{3}$, then $G_{P}$ can be determined directly. In fact, if $P=P_{1}$, then consider the affine part $Z \neq 0$ of $C$, that is, the affine defining equation is $y^{e}-x^{d}=0$. Then, putting $y=t x$, we get $t^{e}-x^{d-e}=0$, hence $G_{P} \cong Z_{d-e}$. The other case $P=P_{2}$ is similarly determined. If $P=P_{3}$, then consider the affine part $X \neq 0$, we get $y^{e} z^{d-e}=1$. Putting $z=t y$, we get $t^{d-e} y^{d}=1$, hence $G_{P} \cong Z_{d}$. As we have seen above, these points are Galois ones.

Next we treat the case $P \in C \backslash\left\{P_{1}, P_{2}\right\}$. First, we prove the subcase $1<e<d-1$. Since $C$ is a self-dual curve and has no flex, we see that, if a line $\ell_{P}$ passes through neither $P_{1}$ nor $P_{2}$, then it has normal crossings with $C$ or it is a simple 2tangent line to $C$. Furthermore, by Hurwitz's formula, we see there exists a simple 2-tangent. Then, by (1) in Proposition 11 and Lemma 15, we have $G_{P} \cong S_{d-1}$. Next we prove the subcase $e=1$. Then, $P_{1}$ (resp., $P_{2}$ ) is a flex (resp., cusp) and the tangent line at $P_{1}$ (resp., $P_{2}$ ) does not meet $C$ except at $P_{1}$ (resp., $P_{2}$ ). If a line $\ell_{P}$ does not pass through $P_{2}$, then it has normal crossings with $C$ or it is a simple 2-tangent line to $C$. By (2) in Proposition 11 and Lemma 15, we have $G_{P} \cong S_{d-1}$. The proof of the case $e=d-1$ is the same.
Now we prove the case where $P \in \mathbb{P}^{2} \backslash C$ and $P \neq P_{3}$. If $P \in \ell_{P_{1} P_{2}}$ and $1<e<d-1$, then $\pi_{P}$ has two ramification points $R_{1}$ and $R_{2}$ such that $e\left(R_{1}\right)=e, e\left(R_{2}\right)=d-e$ and $\pi_{P}\left(R_{1}\right)=\pi_{P}\left(R_{2}\right)$. Thus, $\pi_{P}$ is not an $s$-covering. If $\ell_{P}$ passes through neither $P_{1}$ nor $P_{2}$, then $\ell_{P}$ is a simple 2-tangent to $C$ or has normal crossings with C. By (3) in Proposition 10, $\pi_{P}$ is indecomposable. Since there exists a simple 2 -tangent $\ell_{P}$, we conclude $G_{P} \cong S_{d}$. In case $P \in \ell_{P_{1} P_{2}}$ and $e=1$ or $d-1, \pi_{P}$ is an $s$-covering and $e_{1}=d-1$ and $e_{2}=2$, hence by (2) in Proposition $10, G_{P}$ is primitive and there exists a simple 2 -tangent line $\ell_{P}$, thus we conclude $G_{P} \cong S_{d}$. In view of Remark 19, we conclude easily from the similar argument that $G_{P} \cong S_{d}$ when $P \in \mathbb{P}^{2} \backslash\left\{C \cup \ell_{P_{1} P_{2}}\right\}$.

Proof of the Case (II). Assume $C=C_{(4)}$. It has the following property.

Claim 3. The $T_{P_{1}}$ (resp., $T_{P_{2}}$ ) is $Y=0$ (resp., $Z=0$ ) and $T_{P_{1}} \cap T_{P_{2}}=\left\{P_{3}\right\}$, which does not lie on $C$. Furthermore, $T_{P_{1}} \cap C \stackrel{2}{=}\left\{P_{1}\right\}$ and $T_{P_{2}} \cap C=\left\{P_{2},(1: 1: 0)\right\}$. The $C$ has one flex $F$ of order 1 , that is, $i\left(C, T_{F} ; F\right)=3$ and $T_{F}$ does not pass though $P_{3}$.

Proof. The last assertion is checked by Hurwitz's formula and the others are simple.

Remark 20. The coordinates of the flex $F$ are computed as (-576: $-4096: 135)$.

Clearly, if $P=P_{1}$ or $P_{2}$, then $G_{P} \cong Z_{2}$. If $P \in C \backslash\left\{P_{1}, P_{2}\right\}$, then $n=3$, hence $G_{P}$ is primitive. We divide the proof into three cases:

(1) $P=F$;

(2) $P=(1: 1: 0)$;

(3) $P$ is the other point.

In any case, by Hurwitz's formula, we infer that there exists at least one simple 2-tangent line passing through $P$, hence $G_{P} \cong S_{3}$. Then consider the case $P \in \mathbb{P}^{2} \backslash C$. If $P \in \ell_{P_{1} P_{2}}$, then $\pi_{P}$ has ramification points $R_{1}$ and $R_{2}$ such that $e\left(R_{1}\right)=e\left(R_{2}\right)=2$ and $\pi_{P}\left(R_{1}\right)=\pi_{P}\left(R_{2}\right)$. Thus, $\pi_{P}$ is not an $s-$ covering. Consider $\pi_{P}$ for the most special case $\ell_{P_{1} P_{2}} \cap T_{F}=$ $\{P\}$. We infer from Hurwitz's formula that the ramification data is $\left(3,2^{4}\right):=(3,2,2,2,2)$. By (3) in Proposition 10, we have $G_{P} \cong S_{4}$. There are several cases of position of $P$ which yield different ramification data; however, it is easy to see that there exists $i$ such that $e_{i}=2$. Then from Propositions 10 or 11 , we conclude $G_{P} \cong S_{4}$.

Proof of the Case (III). Assume $C=C_{54}$. It has the following property. There exists a projective transformation $\sigma$ such that $\sigma(C)=C$ and $\sigma(X, Y, Z)=(Y, X,-Z),(-X, Z, Y)$ or $(Z, Y, X)$ so that $\sigma$ interchanges $P_{i}(i=1,2,3)$.

Claim 4. The flexes of $C$ are $F_{1}=(4:-1: 4), F_{2}=(1:$ $-4: 4)$, and $F_{3}=(4:-4: 1)$, hence the tangent lines to $C$ at them are $L_{1}: X+8 Y+Z=0, L_{2}: 8 X+Y-Z=0$, and $L_{3}:-X+Y+8 Z=0$, respectively. On the other hand, the 
tangent lines to $C$ at $P_{1}, P_{2}$, and $P_{3}$ are $L_{4}: X=Y, L_{5}$ : $X=Z$, and $L_{6}: Y=Z$, respectively. There exist just three points $Q_{i}(i=1,2,3)$ satisfying the following conditions:

(1) $Q_{i} \notin C$;

(2) if $\ell=\ell_{Q_{i}}$ does not pass through any cusp, then $\ell$ and $C$ have normal crossings or there exist two points $Q^{\prime} \in$ $C$ satisfying $i\left(C, \ell ; Q^{\prime}\right) \geq 3$.

Such $Q_{i}$ is an intersection $L_{j} \cap L_{k}$, where $\{i, j, k\}=\{1,2,3\}$, indeed $Q_{1}=(1:-7: 1), Q_{2}=(7:-1: 1)$, and $Q_{3}=$ $(1:-1: 7)$. Therefore, if $P \in \mathbb{P}^{2} \backslash\left\{C, Q_{1}, Q_{2}, Q_{3}\right\}$, then there exists a line $\ell$ passing through $P$ such that $\ell$ is a simple 2-tangent line to $C$.

Proof. Making use of the results in [10] and observing the self-duality of $C$, we can check the assertions by direct computations.

Now let us begin the proof. If $P=P_{1}$, then $n=3$, hence $G_{P}$ is primitive. The lines $\ell_{P_{1} P_{2}}$ and $\ell_{P_{1} P_{3}}$ yield the ramification points of order three of $\pi_{P}$, hence we infer from Hurwitz's formula that there exists $i$ such that $e_{i}=2$. Thus, we get $G_{P_{1}} \cong S_{3}$. For $P=P_{2}$ or $P_{3}$, using the projective transformation $\sigma$ above, we see $G_{P_{i}} \cong S_{3}(i=2,3)$.

Next consider the case $P \in C \backslash\left\{P_{1}, P_{2}, P_{3}\right\}$. Then we have $n=5$, hence $G_{P}$ is primitive. Using Hurwitz's formula or the self-duality of $C$, we see that there exists a simple 2-tangent line to $C$, thus we have $G_{P} \cong S_{5}$.

Finally, we consider the remaining case $P \in \mathbb{P}^{2} \backslash C$.

Claim 5. Let $n_{i}$ be the number of ramification points with index $i$. Then we have $n_{2}+2 n_{3}+3 n_{4}=12$, where $n_{4} \leq$ 3. In particular, if $n_{4}=3$ (resp., 2), then $P=(1$ : 1 : 1) (resp., $Q_{i}$ ), furthermore; $n_{3}=0$ (resp., 3) and $n_{2}=$ 3 (resp., 0).

Proof. The former assertion is clear from Claim 4 and Hurwitz's formula. The proof of the latter assertion is as follows: observing Claim 4, we infer that, if $n_{4}=3$, then $P$ is unique $(1: 1: 1)$, which is the intersections of the three lines $L_{4}, L_{5}$, and $L_{6}$ (Figure 1). Similarly observing Claim 4, we infer that if $n_{4}=2$, then $P=Q_{1}, Q_{2}$ or $Q_{3}$. In this case, we have $i\left(C, \ell_{P P_{i}} ; P_{i}\right)=3$, hence $n_{3}=3$.

Claim 6. If $\pi_{P}$ is an $s$-covering, then $\pi_{P}$ is indecomposable.

Proof. By Claim 5 the ramification index is 2, 3, or 4 . Suppose $\pi_{P}$ is decomposable. Then, $\operatorname{deg} \beta=2$ or 3 . By Claim $1, \beta$ is unramified at $R_{i}^{\prime}=\alpha\left(R_{i}\right)$, where $e_{i}=2$ or 3 . By Claim 5 , we have $n_{4} \leq 3$. As we have seen in the proof of Proposition 10, $\beta$ cannot be ramified at only one point. Thus, we have $n_{4} \neq 1$. If $n_{4}=0$, then the proof is clear by (3) in Proposition 11. If $n_{4}=2$, then $P=Q_{i}(i=1,2,3)$. In case $\operatorname{deg} \beta=2$, $\beta$ is ramified at $R_{1}^{\prime}$ and $R_{2}^{\prime}$. Since $\operatorname{deg} \alpha=3$, this cannot occur. In case $\operatorname{deg} \beta=3, \beta$ is ramified at $R_{1}^{\prime}$ and $R_{2}^{\prime}$ with $e\left(R_{1}^{\prime}, \beta\right)=e\left(R_{2}^{\prime}, \beta\right)=2$; however, these do not satisfy Hurwitz's formula. If $n_{4}=3$, then $P=\left(\begin{array}{l}1: 1: 1 \\ \text { : }\end{array}\right.$ and from Claim 4 and Hurwitz's formula we infer that the

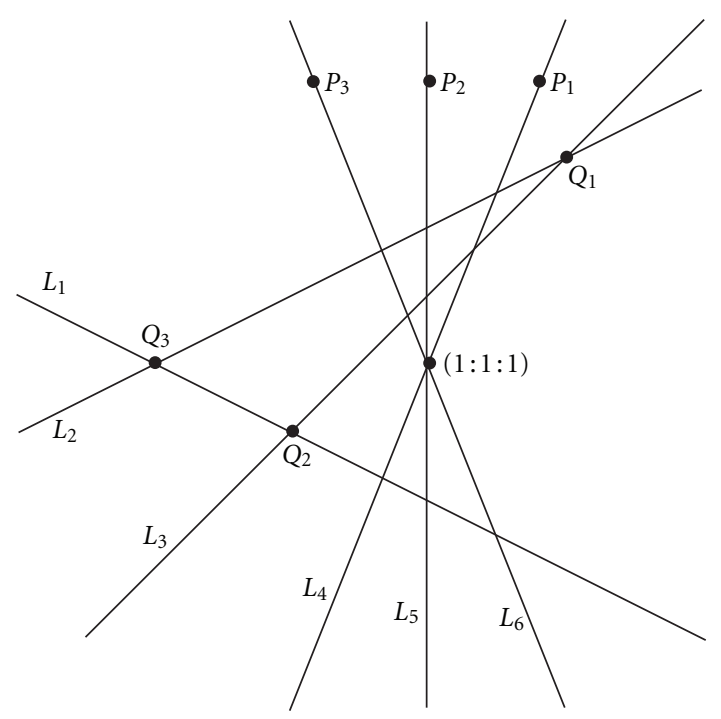

Figure 1

ramification data is $\left(4^{3}, 2^{3}\right):=(4,4,4,2,2,2)$. Suppose $\pi_{P}$ is decomposable. Then, $\operatorname{deg} \beta=2$ or 3 . If $\operatorname{deg} \beta=2$, then $\beta$ is ramified at $R_{i}^{\prime},(i=1,2,3)$. However, since $\operatorname{deg} \alpha=3$, this case cannot occur. Then, we have $\operatorname{deg} \beta=3$. We see easily that $\beta$ is ramified at $R_{i}^{\prime}$ with $e\left(R_{i}^{\prime}, \beta\right)=2(i=1,2,3)$. However, this does not satisfy Hurwitz's formula. Therefore, $\pi_{P}$ is indecomposable.

Now we resume the proof. We prove by examining the cases:

(i) $P=(1: 1: 1)$;

(ii) $P=Q_{i}(i=1,2,3)$;

(iii) $P \in \ell_{P_{i} P_{j}}(1 \leq i, j \leq 3), P \neq(1: 1: 1)$, and $P \neq Q_{i}(i=1,2,3) ;$

(iv) $P$ is the point not appearing in the above case.

By Claims 5 and 6, the proof is complete for (i) and (iv). So let us treat the case (ii). By Claim $6, G_{P}$ is primitive. However, there exists no simple 2-tangent line. Take $Q_{1}=(1:-7: 1)$ and consider the affine part $Z \neq 0$. The defining equation is $(x y-x+y)^{3}+54 x^{2} y^{2}=0$. Putting $u=x-1, v=y+7$ and $v=$ $t u$, we get $h(t, u):=\left(t u^{2}-8 u+2 t u-15\right)^{3}+54(u+1)(t u-$ $7)^{2}=0$. Here, we consider the Galois group obtained by the special value $t=2$. By the aid of a software, for example, PARI, we see that the polynomial $h(2, u)=\left(2 u^{2}-4 u-15\right)^{3}+54(u+$ 1) $(2 u-7)^{2}$ in $\mathbb{Q}[u]$ is irreducible and the Galois group of this polynomial is $S_{6}$. Let $u_{1}(t), \ldots, u_{6}(t)$ be the roots of $h(t, u)=0$ with respect to $u$. Note that $u_{i}(t) \quad(1 \leq i \leq 6)$ is regular near $t=2$ and $\left\{u_{1}(2), \ldots, u_{6}(2)\right\}$ are the roots of $h(2, u)=0$. We can find $c_{i} \in \mathbb{Q}(1 \leq i \leq 6)$ satisfying the conditions: $\tilde{u}(t)=$ $c_{1} u_{1}(t)+\cdots+c_{6} u_{6}(t)$ (resp., $\widetilde{u}(2)=c_{1} u_{1}(2)+\cdots+c_{6} u_{6}(2)$ ) is a generator of the minimal splitting field of $h(t, u)$ (resp., $h(2, u)$ ) over $k(t)$ (resp., $\mathbb{Q}$ ). Suppose the degree of $\widetilde{u}(t)$ is less than 6 !. Then, so is $\widetilde{u}(2)$, which is a contradiction. Hence we have $[k(t, u): k(t)]=6$ !, thus we conclude $G_{P} \cong S_{6}$. The proofs of the other two cases $Q_{2}$ and $Q_{3}$ are almost the same. 
The proof of the case (iii) is as follows: here we notice that if $P \in \ell_{P_{i} P_{j}}, i \neq j,(i, j=1,2,3)$, then $\pi_{P}$ is not an $s^{-}$ covering. First we consider the special case where $P$ is in some $T_{F_{i}}$, for example, $\ell_{P_{1} P_{2}} \cap T_{F_{1}}=\{P\}$. Then the ramification data is $\left\{\left(F_{1}, P_{1}, P_{2}, P_{3}, R_{5}, R_{6}, R_{7}\right),\left(4,3^{3}, 2^{3}\right)\right\}$ and $\pi_{P}\left(P_{1}\right)=$ $\pi_{P}\left(P_{2}\right)$. Suppose $\pi_{P}$ is decomposable. Then, by Claim $1, \beta$ : $X_{3} \rightarrow \mathbb{P}^{1}$ is unramified at $\alpha\left(P_{3}\right)$ and $R_{i}^{\prime},(i \geq 5)$. Namely, $\beta$ is ramified at just two points. Then, the ramification data of $\beta$ is $\left\{\left(\alpha\left(F_{1}\right), \alpha\left(P_{1}\right)\right),(2,2)\right\}$ or $\left\{\left(\alpha\left(F_{1}\right), \alpha\left(P_{1}\right)\right),(3,3)\right\}$, where $\operatorname{deg} \beta=2$ or 3 , respectively. However, it is easy to see that this is impossible considering $\alpha$ and $\pi_{P}$, so $\pi_{P}$ is indecomposable. Since there exist $e_{i}=2(i=5,6,7)$, we conclude $G_{P} \cong S_{6}$. On the other hand, if $P$ is not in $T_{F_{i}}$ for each $i(i=1,2,3)$, then, by (3) in Proposition 10, $f$ is indecomposable. Since there exists a simple 2-tangent, we have $G_{P} \cong S_{6}$.

Thus, we complete all the proofs.

Remark 21. In the list of Theorem 17 only two kinds of group appear. Of course, other kinds will appear in other examples, for example, let us take the Fermat quartic $X^{4}+Y^{4}+Z^{4}=0$. Then, there exist 12 points such that $G_{P}$ is the dihedral group of order 8 (cf. [13]).

Problem. Concerning the Galois groups for $C_{(e, d)}(1<e<$ $d-1)$, full symmetric group $S_{d}$ degenerates into the cyclic group. How does the symmetric group degenerate for various curves?

\section{Acknowledgments}

The authors would like to express their thanks to Oka for teaching the example of self-dual curve $C_{54}$. They thank also the referee(s) for carefully reading the paper and giving the suitable suggestions for improvements.

\section{References}

[1] K. Miura, "Field theory for function fields of singular plane quartic curves," Bulletin of the Australian Mathematical Society, vol. 62, no. 2, pp. 193-204, 2000.

[2] H. Yoshihara, "Function field theory of plane curves by dual curves," Journal of Algebra, vol. 239, no. 1, pp. 340-355, 2001.

[3] H. Yoshihara, "Galois points for plane rational curves," Far East Journal of Mathematical Sciences, vol. 25, no. 2, pp. 273-284, 2007.

[4] H. Yoshihara, "Rational curve with Galois point and extendable Galois automorphism," Journal of Algebra, vol. 321, no. 5, pp. 1463-1472, 2009.

[5] J.-P. Serre, Topics in Galois Theory, vol. 1 of Research Notes in Mathematics, Jones \& Bartlett, Boston, Mass, USA, 1992.

[6] F. Cukierman, "Monodromy of projections," Matemática Contemporânea, vol. 16, pp. 9-30, 1999 (Portuguese), 15th School of Algebra.

[7] J. Harris, "Galois groups of enumerative problems," Duke Mathematical Journal, vol. 46, no. 4, pp. 685-724, 1979.

[8] H. Yoshihara, "Applications of Plücker's formula," Sûgaku, vol. 32, no. 4, pp. 367-369, 1980 (Japanese).

[9] S. Iitaka, K. Ueno, and Y. Namikawa, Descartes No Seishin To Daisûkika, NipponHyoron Sha, Tokyo, Japan, 1980.
[10] M. Oka, "Elliptic curves from sextics," Journal of the Mathematical Society of Japan, vol. 54, no. 2, pp. 349-371, 2002.

[11] G. P. Pirola and E. Schlesinger, "Monodromy of projective curves," Journal of Algebraic Geometry, vol. 14, no. 4, pp. 623-642, 2005.

[12] W. Fulton, Algebraic Curves, Mathematics Lecture Notes Series, Benjamin, New York, NY, USA, 1969.

[13] K. Miura and H. Yoshihara, "Field theory for function fields of plane quartic curves," Journal of Algebra, vol. 226, no. 1, pp. 283-294, 2000. 


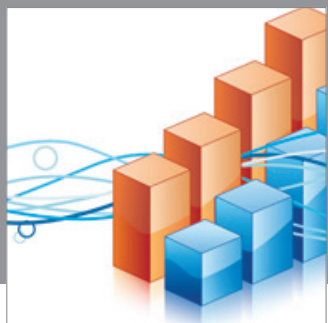

Advances in

Operations Research

mansans

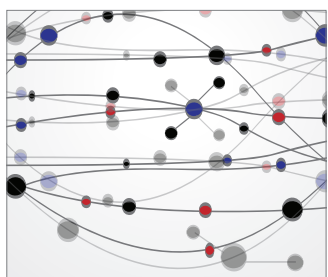

The Scientific World Journal
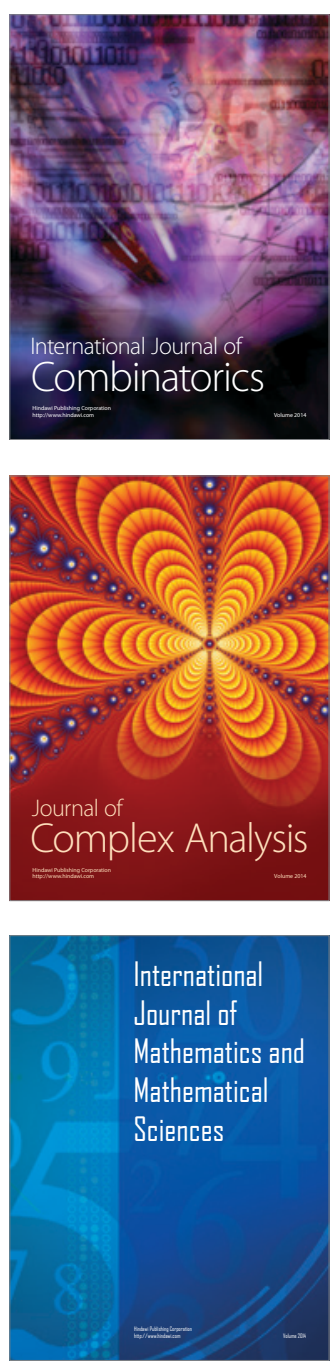


Submit your manuscripts at http://www.hindawi.com
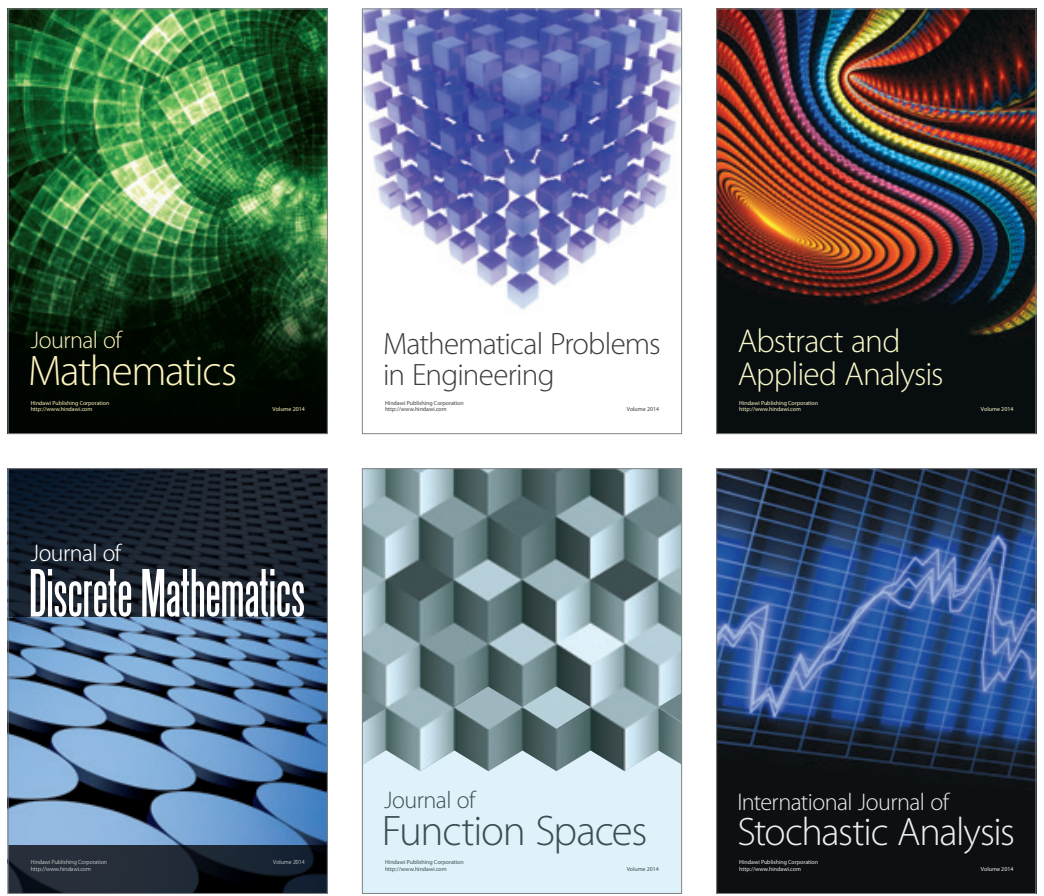

Journal of

Function Spaces




